\title{
THE NONEXISTENCE OF MAXIMAL INVARIANT MEASURES ON ABELIAN GROUPS
}

\author{
ANDRZEJ PELC
}

\begin{abstract}
It is shown that every $\sigma$-additive $\sigma$-finite invariant measure on an abelian group has a proper $\sigma$-additive invariant extension.
\end{abstract}

We consider $\sigma$-finite countably additive measures which vanish on points and are nonidentically zero. Throughout this paper the word "measure" will mean a measure enjoying all the above properties. A measure $m$ defined on a $\sigma$-algebra $S$ of subsets of $X$ is called invariant with respect to a group $G$ of bijections of $X$ if for any $T \in G$ and $A \in S$ the image $T^{*}(A)$ is an element of $S$ and $m\left(T^{*}(A)\right)=m(A)$. A measure $m$ defined on a $\sigma$-algebra of subsets of a group $G$ is called invariant if it is invariant with respect to left translations.

Sierpiński (quoted in Szpilrajn [7]) asked whether there exists in Euclidean $n$ dimensional space $E^{n}$ a maximal extension of the Lebesgue measure invariant with respect to the group of isometries of $E^{n}$. Hulanicki [2] proved that if $|X|$ is less than the first real-valued measurable cardinal, $|G| \leq|X|$, and $m$ is a measure on $X$ invariant with respect to $G$ and vanishing on sets of cardinality $<|X|$, then there exists a proper extension of $m$ invariant with respect to $G$. Thus he solved Sierpiński's problem under additional set theoretic assumptions. Harazišvili [1] gave a negative answer to this question for $n=1$ without any extra hypotheses. He also proved that there is no maximal measure invariant with respect to translations on any Euclidean space. In other words the group of translations of $E^{n}$ does not carry maximal invariant measures. Our theorem is a generalisation of the above result.

THEOREM. Every invariant measure on an abelian group $(G,+)$ has a proper invariant extension.

ProOF. We start with the following lemma, essentially due to Szpilrajn [7]. The easy proof is left to the reader.

LEMMA. Let $m$ be an invariant measure on $G$. If there exists a set $E \subset G$ such that:

1. $E$ is not a set of $m$ measure zero.

2. For every sequence $\left\{g_{n}: n \in \omega\right\}$ of elements of $G$, there exists a sequence $\left\{h_{\alpha}: \alpha<\omega_{1}\right\}$ of elements of $G$ such that for distinct $\alpha, \beta$,

$$
m\left(\left[h_{\alpha}+\bigcup_{n \in \omega}\left(g_{n}+E\right)\right] \cap\left[h_{\beta}+\bigcup_{n \in \omega}\left(g_{n}+E\right)\right]\right)=0
$$

then the measure $m$ has a proper invariant extension.

Received by the editors November 1, 1982.

1980 Mathematics Subject Classification. Primary 28C10, 43A05.

Key words and phrases. Invariant measure, abelian group. 
Hence it suffices to show a set $E$ with the above properties. Without loss of generality we assume that $m$ is a complete measure (i.e. subsets of measure zero sets are measurable).

Case 1. Additive groups of linear spaces over a countable field (cf. Harazišvili [1] and Pelc [4]). Let $V$ be a linear space over a countable field $K$ and $m$ any measure on $V$ invariant with respect to addition. Fix a linear basis $B=\left\{V_{\alpha}: \alpha<\kappa\right\}$ of $V$ over $K$ and let $V_{n}$ denote the set of those elements of $V$ which have $n$ summands in the basis $B$ representation.

Hence $V=\bigcup_{n \in \omega} V_{n}$ and there exists the least number $n_{0}$ for which $V_{n_{0}}$ does not have measure 0 . We claim that $V_{n_{0}}$ also satisfies condition 2 of the lemma.

Let $\left\{g_{n}: n \in \omega\right\}$ be a countable sequence of elements of $V$ and

$$
D=\bigcup_{n \in \omega}\left(g_{n}+V_{n_{0}}\right)
$$

As $\left\{h_{\alpha}: \alpha<\omega_{1}\right\}$ for the lemma take any subset of $B$ of cardinality $\omega_{1}$ whose elements do not appear in the B-representation of $g_{i}-g_{j}$ where $(i, j) \in \omega \times \omega$. Then $w=h_{\alpha}+g_{i}+w_{1}=h_{\beta}+g_{j}+w_{2}$, where $w_{1}$ and $w_{2}$ are in $V_{n_{0}}$, if and only if $g_{i}-g_{j}=h_{\beta}-h_{\alpha}+w_{2}-w_{1}$. Since $h_{\beta}$ and $h_{\alpha}$ are not used in the B-representation of $g_{i}-g_{j}$ and they are distinct, then either $w_{1}=k h_{\alpha}+w^{\prime}$ or $w_{2}=k h_{\alpha}+w^{\prime}$ for some $k \in K$ and $w^{\prime}$ in $V_{n_{0}-1}$. Hence $w=k^{\prime} h_{\alpha}+g_{i}+w^{\prime}$ or $w=h_{\beta}+g_{j}+k^{\prime} h_{\alpha}+w^{\prime}$ for some $k^{\prime} \in K$ and $w^{\prime} \in V_{n_{0}-1}$ so that for $\alpha \neq \beta$ the set $\left(h_{\alpha}+D\right) \cap\left(h_{\beta}+D\right)$ is a subset of a countable union of translations of $V_{n_{0}-1}$. Therefore $\left(h_{\alpha}+D\right) \cap\left(h_{\beta}+D\right)$ has $m$ measure zero. Hence the set $V_{n_{0}}$ satisfies the conditions of the lemma.

Case 2. Torsion-free abelian groups. Let $G$ be a torsion-free abelian group. There exists a homomorphic embedding of $G$ into the additive group of a linear space $V$ over the field $Q$ of rationals such that a certain basis $B=\left\{v_{\alpha}: \alpha<\kappa\right\}$ of $V$ consists of elements of $G$. Let $m$ be any invariant measure on $G$.

For any finite sequence $s=\left(q_{1}, \ldots, q_{n}\right)$ of nonzero rationals let $V_{s}$ be the set of elements of $V$ of the form $q_{1} v_{\alpha_{1}}+\cdots+q_{n} v_{\alpha_{n}}$ where $\alpha_{1}>\cdots>\alpha_{n}$ and $v_{\alpha_{i}} \in B$. Let $s_{0}=\left(r_{1}, \ldots, r_{n}\right)$ be a sequence for which the set $E=G \cap V_{s_{0}}$ is not a set of $m$ measure 0 . In order to check that $E$ also satisfies condition 2 of the lemma, let $\left\{g_{n}: n \in \omega\right\}$ be any sequence of elements in $G$. Take any uncountable set of elements $w_{\alpha}$ of $B$ which do not appear in the B-representation of any element $g_{n}$. Let $k$ be a natural number different from all $r_{i}, r_{i}-r_{j}(i, j \leq n)$ and $h_{\alpha}=k w_{\alpha}$ for $\alpha<\omega_{1}$. We claim that

$$
\left[h_{\alpha}+\bigcup_{n \in \omega}\left(g_{n}+E\right)\right] \cap\left[h_{\beta}+\bigcup_{n \in \omega}\left(g_{n}+E\right)\right]=\varnothing .
$$

Indeed, suppose $x$ is an element of the set on the left side. Then

$$
x=k w_{\alpha}+g_{n}+r_{1} v_{\alpha_{1}}+\cdots+r_{n} v_{\alpha_{n}}=k w_{\beta}+g_{m}+r_{1} v_{\beta_{1}}+\cdots+r_{n} v_{\beta_{n}} .
$$

Since $\alpha \neq \beta$ and $w_{\alpha}, w_{\beta}$ do not appear in the representation of $g_{n}, g_{m}$, we get that either $k=r_{i}$ or $k+r_{i}=r_{j}$ for some $i, j \leq n$, contradiction.

Case 3. Arbitrary grpups. Let $G$ be an arbitrary abelian group and $m$ an invariant measure on $G$. By $H$ denote the torsion subgroup of $G$. If $m(H)=0$ we define a measure $m_{1}$ on $G / H$, putting $m_{1}(\{a+H: a \in A\})=m\left(\bigcup_{a \in A}(a+H)\right)$ 
for $A \subset G$ such that $\bigcup_{a \in A}(a+H)$ is $m$-measurable. The measure $m_{1}$ is clearly invariant (and vanishes on points since $m(H)=0$ ). The group $G / H$ is torsion-free and, hence, by Case 2 there exists a set $E_{1} \subset G / H$ satisfying both conditions from the lemma for $G / H$ and $m_{1}$. It is not hard to see that the set $E=\bigcup E_{1}$ satisfies the conditions from the lemma for $G$ and $m$.

If $H$ is not a set of $m$ measure 0 then let $H_{n}$ (for $n \geq 1$ ) denote the subgroup of $H$ consisting of those elements whose orders divide $n$. Clearly $H=\bigcup_{n \geq 1} H$ and let $n_{0}$ be the least natural number for which $H_{n_{0}}$ is not a set of $m$ measure 0 . We will prove the existence of a subset of $H_{n_{0}}$ satisfying the conditions of our lemma by induction on the number of prime divisors of $n_{0}$ (counting multiple divisiors many times). If $k=1$ then $n_{0}$ is prime and $H_{n_{0}}$ is the additive group of a linear space over the field $F_{n_{0}}$. Next we proceed as in Case 1 and show that the set constructed there is as required (for $G$ and $m$ ).

Suppose that for $n_{0}$ having $k$ prime divisors there exists a set $E \subset H_{n_{0}}$ satisfying the lemma. Now let $n_{0}=p_{1} \cdots p_{k+1}\left(p_{i}\right.$-primes, $\left.k \geq 1\right)$ and let $H^{\prime}$ be the subgroup of $H_{n_{0}}$ consisting of elements of order $p_{1}$. Since $m\left(H^{\prime}\right)=0$, we can define an invariant measure $m^{\prime}$ on $G / H^{\prime}$ just as before. $H_{n_{0}} / H^{\prime}$ is a subgroup of $G / H^{\prime}$ all of whose elements have orders dividing the number $p_{2} \cdots p_{k+1}$. By definition $H_{n_{0}} / H^{\prime}$ is not a set of $m^{\prime}$ measure 0 . Hence by the inductive hypothesis there exists a set $E^{\prime} \subset H_{n_{0}} / H^{\prime}$ which satisfies the conditions of the lemma for the group $G / H^{\prime}$ and measure $m^{\prime}$. It is easy to see that set $E=\bigcup E^{\prime}$ is now good for $G$ and $m$, which finishes the proof in the general case.

\section{REFERENCES}

0. P. Erdös and R. D. Mauldin, The nomexistence of certain invariant measures, Proc. Amer. Math. Soc. 59 (1976), 321-322.

1. A. B. Harazišvili, On Sierpiński's problem concerning strict extendibility of an invariant measure, Soviet Math. Dokl. 18 (1977), 71-74.

2. A. Hulanicki, Invariant extensions of the Lebesgue measure, Fund. Math. 51 (1962), 111-115.

3. A. Pelc, Semiregular invariant measures on abelian groups, Proc. Amer. Math. Soc. 86 (1982), 423-426.

4. - The nonexistence of maximal invariant extensions of the Lebesgue measure, preprint.

5. __ Invariant measures and ideals on discrete groups (to appear).

6. C. Ryll-Nardzewski and R. Telgarsky, The nonexistence of universal invariant measures, Proc. Amer. Math. Soc. 69 (1978), 240-242.

7. E. Szpilrajn, Sur l'extension de la mesure lebesguienne, Fund. Math. 25 (1935), 551-558.

Department of Mathematics, University of WarsaW, PKin IXP, 00-901, WaRSAW, POLAND 